\title{
Contributions to the contact resistivity in fired tunnel-oxide passivating contacts for crystalline silicon solar cells
}

\author{
F.-J. Haug ${ }^{1}$, A. Ingenito ${ }^{1}$, F. Meyer ${ }^{1}$, S. Libraro ${ }^{1}$, N. Bolis ${ }^{1}$, J.J. Diaz Leon ${ }^{2}$, C. Allebé ${ }^{2}$, C.
} Ballif ${ }^{1,2}$

${ }^{1}$ Ecole Polytechnique Fédérale de Lausanne (EPFL), PV-Lab, Maladière 71b, CH-2000 Neuchâtel

${ }^{2}$ Centre Suisse d'Electronique et Microtechnique (CSEM), PV-Centre, Jaquet-Droz 1, CH-2000

Neuchâtel

\begin{abstract}
We investigate the contact resistivity of $p$ type passivating contacts for silicon solar cells. Our contact structures are compatible with firing, a rapid annealing process similar to the one used for sintering of the screen-printed metallisation in solar cell manufacturing. We find that the short firing process crystallises the doped layers and incorporates active boron dopants up to the solubility concentration at the chosen firing temperature. The dependence of our contact resistivities on carrier density and temperature suggest that the hole transport is a combination of tunnelling through the oxide at the wafer surface and of thermionic field emission over the Schottky barrier to the metallisation. For ideal firing conditions we find implied open circuit voltages up to $720 \mathrm{mV}$ and contact resistivities as low as $15 \mathrm{~m} \Omega \mathrm{cm}^{2}$.
\end{abstract}

Index Terms - contact resistivity, passivating contact, silicon solar cell

\section{INTRODUCTION}

Carrier selective passivating contacts are a key ingredient for high efficiency silicon solar cells. Conversion efficiencies up to $26.7 \%$ were recently demonstrated for back contacted $n$-type cells, using a low-temperature heterojunction with amorphous silicon passivating layers [1], [2]. Alternatively, a high-temperature approach with a tunnel-oxide passivating contact (TOPCon) on $n$-type wafers led to efficiencies of up to $25.7 \%$ [3]. For a passivating contact of poly-Si on oxide (POLO) in a rear contacted architecture on $p$-type wafers, an efficiency of $26.1 \%$ was demonstrated [4]. Despite the impressive performance of passivating contacts, a rapid transfer to manufacturing has been hampered by various aspects. For example, rear contacted cells usually require an elaborate processing sequence with various lithographic and aligning steps, and a process without lithography was shown only recently [5]. The implementation of the less commonly used $n$-type wafers in some of the approaches was also considered a hindrance, but this fear may yet be proven wrong since the production of $n$-type cells with tunnel oxide passivating contacts are currently scaled up to the GW level [6]. Another hindrance that also applies to $p$-type silicon solar cells and thus to a large majority of silicon solar cells, is their formation by long annealing processes at high temperatures. A possible solution to this issue was the recent demonstration of $p$-type fired passivating contacts (FPCs) since they can be processed with conditions that are compatible with the rapid thermal annealing step used in large-scale manufacturing for sintering of the metallisation [7].

Owing to dwell times of only a few seconds at typical firing temperatures between 750 and $850^{\circ} \mathrm{C}$, the thermal budget of FPCs is not sufficient to diffuse dopants across the oxide into the region close to the wafer surface [7]. Instead, the highly doped contact layer induces an accumulation layer across the passivating oxide into the wafer. Therefore, a careful passivation of structural defects in the thin oxide layer and at its interfaces is needed to avoid trapping of charge carriers and thus a reduction of the band bending $(\mathrm{BB})$ in the accumulation layer.

In this contribution, we analyse the doping concentration in the contact layer after firing, we characterise the contact resistivity between the wafer 
and the external metallisation, and we discuss possible current transport mechanisms.

\section{EXPERIMENTAL}

Our experiments were carried out on boron-doped $<100\rangle$ float zone wafers with a thickness of $200 \mu \mathrm{m}$ and a resistivity of $2 \Omega \mathrm{cm}$. A measurement of the shiny etched (SE) surface finish with AFM (not shown) revealed an RMS roughness of ca. $15 \mathrm{~nm}$. After cleaning, both sides were covered with a ca. $1.2 \mathrm{~nm}$ thick oxide, either by immersion into hot $\mathrm{HNO}_{3}(69 \%$, $80^{\circ} \mathrm{C}$ ) or by ozone under UV light of a mercury lamp (Jelight, UVO cleaner42). Plasma enhanced chemical vapour deposition (PECVD) with an excitation frequency of $40.6 \mathrm{MHz}$ was used to grow $40 \mathrm{~nm}$ thick p-type $\mathrm{SiC}_{\mathrm{x}}: \mathrm{B}$ on both sides of the $\mathrm{Si}$ wafer at $200^{\circ} \mathrm{C}$, using silane $\left(\mathrm{SiH}_{4}\right)$, hydrogen $\left(\mathrm{H}_{2}\right)$, methane $\left(\mathrm{CH}_{4}\right)$ and tri-methyl-boron $\left(\mathrm{B}\left(\mathrm{CH}_{3}\right)_{3}, \mathrm{TMB}\right)$. Subsequently, the samples were fired by rapid thermal processing (RTP) in argon atmosphere using fast ramping of $50^{\circ} \mathrm{C} / \mathrm{s}$ and a dwell time of 3 seconds at temperatures between 770 and $830^{\circ} \mathrm{C}$ (Jetfirst 200, Jilpelec). For the firing experiments, the temperature was controlled by a pyrometer against reference measurements with thermocouples in contact to the wafer. Finally, the samples were hydrogenated by applying a layer of SiNx by PECVD on both sides and annealing in a firing furnace in air (Camini, Meyer-Burger). We estimate dwell times of about $3 \mathrm{~s}$ from the speed of the transport rollers at set temperatures between 770 and $830^{\circ} \mathrm{C}$. Since the temperatures are controlled by thermocouples in the heating zones, we estimate that the actual sample temperatures are 60 to $70^{\circ} \mathrm{C}$ lower, i.e. less than the firing temperatures. After hydrogenation, the SiNx layers are stripped in $\mathrm{HF}$.

Optical characterisation was carried out with ellipsometry (UVISEL, Horiba Jobin Yvon), UV-Vis spectrometry (Lambda 950 with integrating sphere, Perkin Elmer), and IR transmission (Vertex 90 FTIR, Bruker). The contact resistivity was characterised by the transfer length method (TLM). Samples were dipped in HF to remove native oxides, then an ITO/Ag bilayer was sputtered through openings in a shadow mask. This contact structure was chosen because it is also used in our demonstrator solar cells. Subsequently, the samples were cleaved along the TLM pattern to avoid lateral current spreading. The current spreading into the wafer was corrected according to ref. [8]. Since sputtering can deposit material under the shadow mask, the true distances between the pads was measured by optical microscopy. The area between the pads was not etched since the sheet resistance of the fired layer is negligible with respect to the wafer as discussed below. The shown results are averages over three individual samples, the error bars denote their standard deviation.

The implied open circuit voltage $i V_{o c}$ of the fired passivating contacts was determined on the symmetric samples by photconductance decay (Sinton WTC 120).

\section{OPTICAL CHARACTERISATION}

The carrier density in the crystallised film is of interest for a variety of reasons; first, it will impact the contact resistivity $\rho_{c}$ to the external metallisation, in our case ITO. Second, in the absence of an in-diffused region it governs the induced accumulation layer. Third, it may reduce the photocurrent by parasitic absorption. Since our sample structures is $\mathrm{p}^{+} / \mathrm{p} / \mathrm{p}^{+}$, we cannot characterise it by Hall measurement. Alternatively, it can be assessed by its free carrier absorption (FCA).

\section{A. Free carrier absorption}

The FCA of in a thin film can be measured by IR transmission [9]. The impact of light scattering at the surface morphology of the SE wafers against a double side polished wafer (DSP) with a thickness of $280 \mu \mathrm{m}$. The transmission spectra of the DSP and the SE wafers in Fig. 1 show that light scattering becomes negligible below $0.2 \mathrm{eV}$ because the wavelength in this spectral region is above $6 \mu \mathrm{m}$ and thus more than 500 times larger than the surface roughness. The transmission data of bulk silicon was modelled on the basis of published permittivity data for the visible [10] and for the IR [11], [12]. Nevertheless, we note that the method is not applicable to wafers with deeper textures such as a saw-damage etch or random pyramids.

The visible and UV properties were measured by ellipsometry between 0.6 and $6 \mathrm{eV}$ in reflection geometry under an angle of $70^{\circ}$. Above the bandgap energy of silicon, ellipsometry probes the primary reflection and thus the moderate surface roughness of the SE samples has a minor impact. Discrepancies were found for energies below the bandgap where the samples become transparent. Due to a small spot size and light scattering within the wafer, the contribution from the rear surface is collected only partially, and consequently the fitting of the ellipsometry data was restricted to the region between 1.2 and $6 \mathrm{eV}$. The deviations between measured data and fitted curves are shown in the inset of Fig. 1 for energies below the bandgap. 
To obtain more reliable data in the spectral region around the onset of transparency, we measured the total transmission between 0.5 and $1.4 \mathrm{eV}$ in a spectrometer with integrating sphere. Finally, the signature of FCA was assessed by the IR transmission at photon energies between 0.05 and $0.15 \mathrm{eV}$. Fig. 1 shows that this region lies outside of the absorption bands of ambient influences, especially $\mathrm{H}_{2} \mathrm{O}$. The data was modelled with the software package DeltaPsi (Horiba Jobin Yvon), using a bound model to simultaneously fit all three spectral regions with the same model.

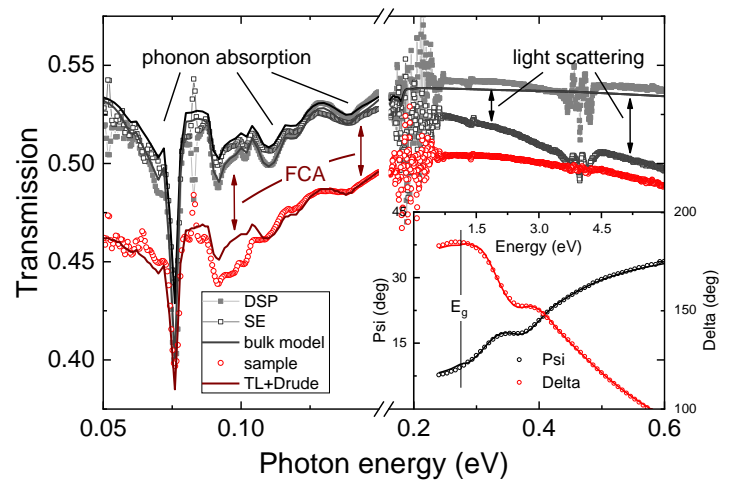

Fig. 1: IR transmission spectra of DSP and SE wafer (full and open squares, respectively) and of a sample with a doped $\mathrm{SiC}_{\mathrm{x}}$ layer on both sides (circles, measured after firing at $770^{\circ} \mathrm{C}$ ). Overlaid lines denote modelling results. The inset shows ellipsometry data (symbols) and fitting results (lines) of the same sample.

The crystallized $\mathrm{SiC}_{\mathrm{x}}$ layer was described with a combination of a Tauc-Lorentz model and of a Drude model. The former uses the parameters $n_{\infty}$ (refractive index), $E_{g}$ (bandgap), $A$ (amplitude), $E$ (resonance energy), and $C$ (damping constant), the latter uses the parameters $\omega_{p}$ (plasma frequency) and $\Gamma$ (damping constant). Since we used symmetric samples, we constrained the seven parameters of the layer, and its thickness and its surface roughness to assume the same values on the front and on the rear.

\section{B. Dopant activation}

We determined a first guess for the free carrier density and the optical mobility from $\omega_{p}$ and $\Gamma$, respectively, using a value of $m_{h}^{o p t}=0.4 m_{e}$ for the optical effective mass [13], [14]. The value of the carrier density was then used to estimate the concentration profile of the induced accumulation layer for the lowest and highest carrier densities of our sample set. Even for the ideal assumption of zero trapped charge in the oxide layer and at its interfaces, the induced carrier density is less than $10 \%$ of the carrier density in the fired layer, and it decays to the bulk doping over a typical length-scale that is less than the thickness of the $\mathrm{SiC}_{\mathrm{x}}$ layer. Consequently, we decided to continue with the simple optical model rather than refining it with the contribution of the accumulation layer.

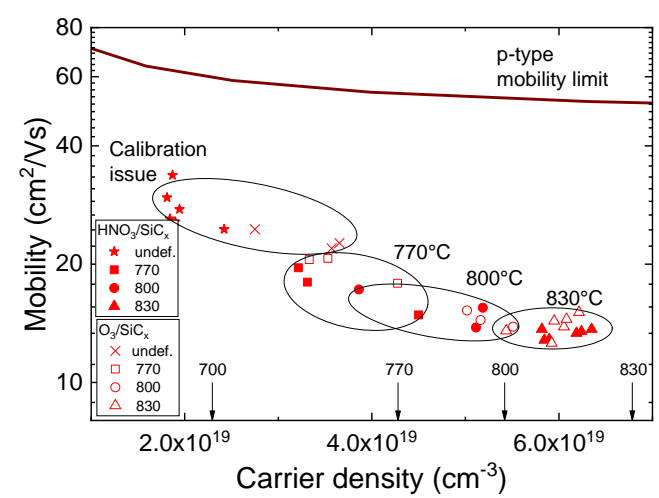

Fig. 2: Optical mobility vs. carrier density of $\mathrm{SiCx}$ :B layers fired at different temperatures. Stars refer to undefined firing temperatures due to a calibration error. Arrows denote the saturation concentrations of B in Si at the indicated temperatures [15], the line illustrates the hole mobility limit due to ionised impurity scattering in $\mathrm{Si}[16]$.

Fig. 2 shows that increasing firing temperatures increase the carrier densities and decrease the optical mobilities. The temperature of the hydrogenation step does not show an influence, corroborating our assumption that it does not exceed the firing temperatures. For firing at $770^{\circ} \mathrm{C}$ and $800^{\circ} \mathrm{C}$, the carrier densities show some scatter, but the respective upper limits agree well with the corresponding solubility limit of boron in bulk $\mathrm{Si}$, marked by the black arrows above the abscissa [15], [17]. For $830^{\circ} \mathrm{C}$ the values scatter less, but they remain slightly below the solubility limit. Carrier density and mobility do not appear to depend on the type of tunnelling oxide, suggesting that the observed changes relate only to crystallisation of the films at the respective firing temperatures.

The figure includes a set of samples for which a calibration issue resulted in low firing temperatures. Even though Raman measurements suggest that these samples contain a large amorphous fraction, they appear to continue a trend of increasing optical mobility for decreasing carrier density.

Over the whole set of samples, the relation between optical mobility and carrier density resembles the behaviour of ionised impurity scattering, but the values in the crystallised layers are significantly lower than the limiting mobility of bulk silicon [16]. Since 
all samples were prepared with the same mix of precursor gases, we conclude that a large ratio of dopants is inactive in the crystallised films, possibly by being incorporated into the amorphous matrix or at the surface of the crystallites.

\section{CONTACT RESISTIVITY}

Fig. 3 shows the variation of contact resistivities with respect to the carrier density in the crystallised layers. Most of the samples fired at temperatures between 800 and $830^{\circ} \mathrm{C}$ lie between 20 and $50 \mathrm{~m} \Omega \mathrm{cm}^{2}$. For typical photocurrents and open circuit voltages in silicon solar cells, we can estimate that the corresponding losses in the fill factor are less than $0.3 \%$ relative for full area coverage [18]. There is no clear dependence of the measured contact resistivities on the type of interfacial oxide, but for high carrier densities the $\mathrm{O}_{3}$ oxides appear to appear to yield somewhat higher values than the $\mathrm{HNO}_{3}$ oxides. This would corroborate the finding that $\mathrm{O}_{3}$ oxides are denser and more stoichiometric than $\mathrm{HNO}_{3}$ oxides [19], [20].

Since we study $\mathrm{p}^{+} / \mathrm{p} / \mathrm{p}^{+}$structures we have to clarify the path of the current in the TLM measurement. To this end, we have to compare the wafer sheet resistance of ca. $100 \Omega$ /square to the one of the crystallised layer. From the layer thickness of ca. $40 \mathrm{~nm}$ and the values of the carrier density and the optical mobility in Fig. 2 we project sheet resistances above $1.5 \mathrm{k} \Omega$ /square, not yet taking into account additional resistance due to grain boundary scattering. Accordingly, the current transport between the TLM pads will take place almost entirely in the wafer. By the same line of argument we find that resistivity due to the transverse transport through the film is orders of magnitude below the values shown in Fig. 3. The contact resistivity $\rho_{c}$ is therefore described by a series connection of the Schottky junction at the $\mathrm{SiC}_{\mathrm{x}} / \mathrm{ITO}$ interface and of the tunnelling junction at the wafer/ $/ \mathrm{SiO}_{\mathrm{x}} / \mathrm{SiC}_{\mathrm{x}}$ interface. The properties of these two junctions are discussed in the following two sections.

\section{A. Tunnel oxide junction}

The prevailing transport process of the tunnel-oxide junctions used in solar cells depends on the processing conditions; experimental evidence suggests transport through pinholes that form in the oxide for samples annealed at high temperatures and tunnelling through a continuous oxide layer for samples annealed with low thermal budget [21], [22]. For our samples we assume that the tunnelling process prevails since the firing temperatures did not exceed $830^{\circ} \mathrm{C}$ and lasted only for a few seconds.

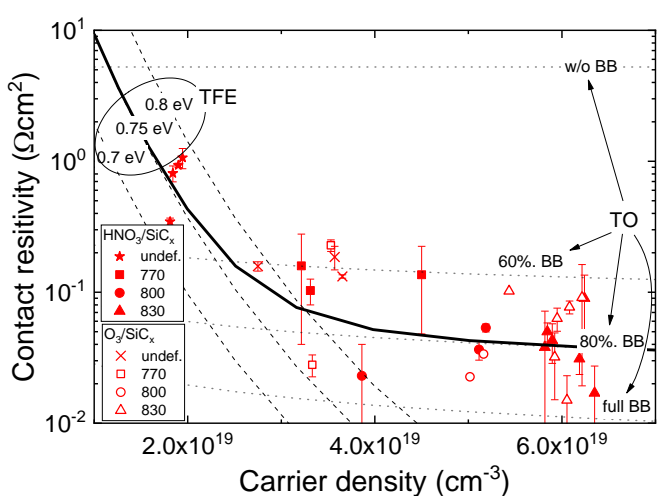

Fig. 3: Contact resistivity vs. carrier density, error bars denote the standard deviation over three samples. Dotted lines illustrate tunnelling transport across an oxide barrier for the shown extent of the band bending. Dashed lines illustrate the contact resistivity expected for thermionic field emission (TFE), using the shown values for the Schottky barrier. The full line illustrates the sum of both transport processes for intermediate parameters.

Accordingly, in Fig. 3, we added characteristics expected for tunnelling junctions [23], using a thickness of $1.2 \mathrm{~nm}, m_{t}^{o x}=0.25 m_{e}$ for the tunnelling mass of holes in the oxide, and $4.8 \mathrm{eV}$ for the barrier height [24], [22]. The shown characteristics vary in the extent of band bending that the crystallised layer induces across the oxide. The lower limit is zero (flatband condition), the upper limit represents the optimum case without charge in the oxide or at the interfaces. Our measurements fall between these extremes, but they are closer to the latter situation.

\section{B. Schottky junction}

We modelled the contribution of the Schottky junction for the cases of thermionic field emission (TFE) and pure field emission (FE) [25], using $m_{h}^{\sigma}=0.66 m_{e}$ for the conductivity effective mass [26]. Fig. 3 shows that this would suggest barrier heights between 0.7 and 0.8 $\mathrm{eV}$ for the samples with low carrier density. For intermediate carrier densities, an agreement between the TFE model and the measured values would require even higher barriers. In the region of the highest shown carrier densities the FE model should be applied, but it predicts $\rho_{c}$ values even lower than the TFE model.

Fig. 3 includes a sum of the two contributions, using intermediate values for both transport parameters. To better clarify the underlying transport properties we measured the temperature dependence of $\rho_{c}$ for three different carrier densities as shown in Fig. 4. In the two samples with low doping $\rho_{c}$ decreases with increasing 
temperature, for the most highly doped sample there is no systematic temperature dependence. Overlaid are modelled characteristics that sum the contribution of TFE with a barrier of $0.7 \mathrm{eV}$ and TO transport. The band bending $(\mathrm{BB})$ that enters the latter characteristic was changed to assume $60 \%, 90 \%$ and $100 \%$ of the ideal value for low, intermediate and high carrier densities, respectively. Whereas the measured temperature dependences are too low for pure TFE in all cases, a transition between TFE and TO could tentatively explain the behaviour of the samples with low carrier density whereas the sample with high carrier density appears to be dominated by TO transport.

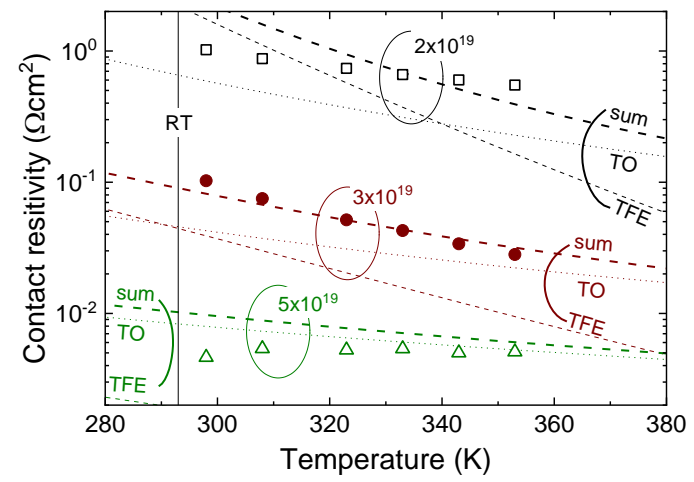

Fig. 4: Temperature dependence of selected samples with $\boldsymbol{N}_{\boldsymbol{A}}=$ $2 \times 10^{19} \mathrm{~cm}^{-3}$ (black squares), $3 \times 10^{19} \mathrm{~cm}^{-3}$ (red circles), and $5 \times 10^{19} \mathrm{~cm}^{-3}$ (green triangles). Closed and open symbols refer to $\mathrm{HNO}_{3}$ and $\mathrm{O}_{3}$ oxides, respectively. Thin dashed and dotted lines illustrate the contributions by TFE and TO transport, respectively, the thick lines represent their sum.

\section{DISCUSSION}

The measurements presented in the previous section suggest that the contact resistivity of our samples is a combination of the tunnelling transport through the interfacial oxide between the wafer and the p-type $\mathrm{SiC}_{\mathrm{x}}$ layer, and of the Schottky barrier between the $\mathrm{p}$ type $\mathrm{SiC}_{\mathrm{x}}$ layer and the n-type ITO layer of the metallisation. In Fig. 5 we propose a band diagram that incorporates these findings.

\section{A. Tunnelling transport}

The tunnelling current depends on the properties of the barrier such as its width and its height, and on the supply function, i.e. the carrier density at the interface. The upper limit of the contact resistivity is thus associated with a case where the carrier density is equal to the bulk doping of the wafer, i.e. a flat-band condition. Lower contact resistivities are expected when the high carrier density of the crystallised layer can induce an accumulation layer across the oxide.
Provided the oxide is thin enough to permit Fermilevel alignment between the wafer and the fired layer, the band bending can be determined by solving the Poisson equation. The maximum extent of $\mathrm{BB}$ is then associated with the case where the oxide and the interfaces do not contain any trapped charge. For an oxide thickness of $1.2 \mathrm{~nm}$, we find for our range of carrier densities that the upper limit of the BB varies from 130 to $160 \mathrm{meV}$. Fig. 3 shows that this assumption results in contact resistivities just below the best of our samples. Most of our data is centred around a model where the BB is reduced empirically to $80 \%$ of this limit and only the samples with low doping concentration exceed the characteristic corresponding to $60 \%$ of the BB. The simplest possibility to account for such a reduction in $\mathrm{BB}$ would be an interface charge due to trapping of holes in defect states at the interface between the wafer and the oxide. For a sample with a carrier density of $5 \times 10^{19} \mathrm{~cm}^{-3}$ in the crystallised layer, we estimate that the ideal band bending is ca. $0.155 \mathrm{eV}$ and reductions to 80 and $60 \%$ would correspond to trapped charge densities of ca. $4 \times 10^{11}$ and $6 \times 10^{11} \mathrm{~cm}^{-2}$, respectively. These estimates are two to three times higher than typical $Q_{i t}$ reported for the $\mathrm{Si} / \mathrm{SiO}_{2}$ interface [27], but considering that the cited values were measured on thick thermal oxides, our estimate for the low temperature oxides does not appear unreasonable.
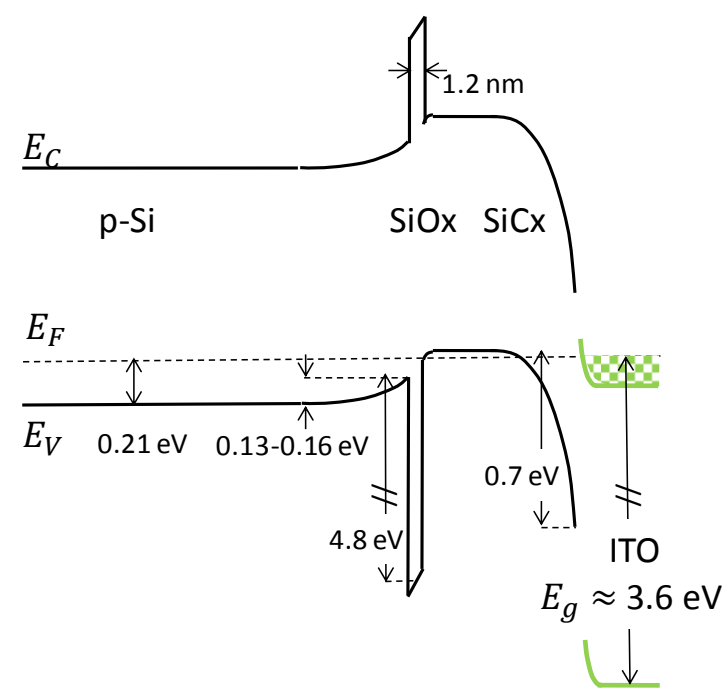

Fig. 5: Proposed band diagram for a fired p-type passivating rear contact.

For a flat-band situation we can estimate an interface charge density of $1.7 \times 10^{12} \mathrm{~cm}^{-2}$ by requiring a voltage drop of $0.21 \mathrm{~V}$ across the interfacial oxide. Recently, a case close to a flat-band condition with very high contact resistivity was reported for a junction very 
similar to ours [22]. There are nevertheless several distinguishing aspects. First, the authors used a thicker interfacial layer of $1.7 \mathrm{~nm}$. Second, they report a carrier density of $5 \times 10^{19} \mathrm{~cm}^{-3}$ after annealing at $700^{\circ} \mathrm{C}$ which is about double the value we would expect from the saturation concentration of boron in silicon as shown in Fig. 2. Third, they use a dwell time of $30 \mathrm{~min}$ to obtain full crystallisation, but they do not comment whether they re-introduced hydrogen after the annealing. Finally, they used an Al metallisation whose Schottky barrier is likely lower than the one of our ITO contacts.

\section{B. Schottky barrier}

With an electron affinity of $4.05 \mathrm{eV}$ and a bandgap of $1.12 \mathrm{eV}$, the valence band (VB) of silicon is $5.17 \mathrm{eV}$ below the vacuum level. For the range of carrier densities in our samples, the Fermi-level varies from a positon of $-15 \mathrm{meV}$ within the gap to a degenerate condition of $+35 \mathrm{meV}$ within the VB.

Data on the work function of ITO scatters widely; values between 4.8 and 4.9 were reported in a study that covered the whole composition range between $\mathrm{In}_{2} \mathrm{O}_{3}$ and $\mathrm{SnO}_{2}$ [28], whereas values between 4.4 to $4.5 \mathrm{eV}$ were reported for an $\mathrm{In} / \mathrm{Sn}$ ratio of ca. 6 with very little dependence on surface- or heat treatment [29]. For an In/Sn ratio of ca. 10, a systematic increase from 4.5 to $5.2 \mathrm{eV}$ was reported for increasing oxygen partial pressure during deposition [30].

Upon contact formation, a Schottky barrier of $0.75 \mathrm{eV}$ would comply with the lowest ITO work functions of the cited range. However, the silicon surface is prone to the formation of surface defects [31] and interfacial states that give rise to Fermi-level pinning [32]. Moreover, it was reported that high values for the work function of ITO may be related to surface band bending rather than to changes in the bulk of the material [30]. Thus, the Schottky barrier implied by our contact resistivities might be the result of an interfacial dipole. It is also possible that the presence of an amorphous phase increases the contact resistivity by current crowding into the crystalline parts of the layer, especially in the samples with low carrier density.

\section{APPLICATION}

We finish by briefly discussing the passivation that these contacts provide to the wafer surface. Fig. 6 collects the implied open circuit voltage $i V_{o c}$ for all shown samples. Whereas the previous sections showed that type of interfacial oxide has no influence on the carrier density and the mobility, and only a minor impact on the contact resistivity, $\mathrm{O}_{3}$ grown oxides generally provide better passivation than $\mathrm{HNO}_{3}$ oxides. In the best case we find an $i V_{o c}$ of $720 \mathrm{mV}$, the same sample has a contact resistivity of $90 \mathrm{~m} \Omega \mathrm{cm}^{2}$ which is still acceptable for a full area contact. We will report elsewhere on the fabrication of complete solar cells using a single firing step for the formation of a passivating SiCx rear contact and the contacting of a diffused emitter with SiNx:H anti-reflection coating at the front.

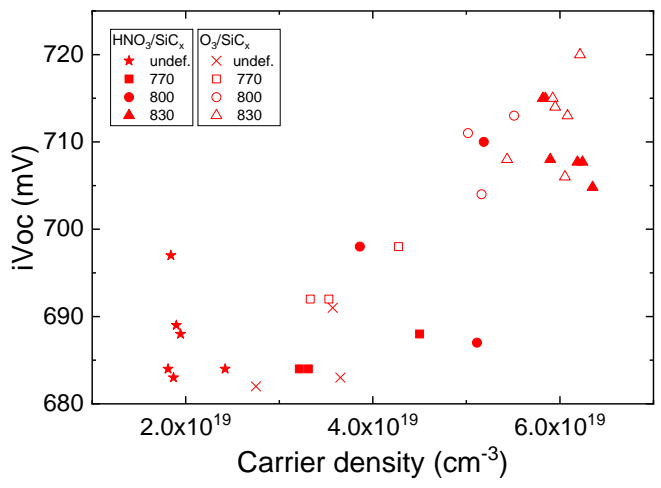

Fig. 6: Implied Voc vs. carrier density.

\section{CONCLUSIONS}

We presented a method to characterise the carrier density and the optical mobility in the layer stacks that are used for passivating contacts in silicon solar cells. Other than Hall measurements, our method can be applied to isotype junctions such boron doped films on $p$-type wafers. We found that the carrier density increases with the chosen firing temperature and that the attained values are consistent with the solubility limit at the respective temperatures. The mobilities decrease with increasing carrier density, reminiscent of ionised impurity scattering, but attaining values much below the bulk mobility limit for this scattering mechanism. Likely, the difference is due to the presence of inactive dopants and the effect of grain boundary scattering cannot be excluded, especially for the samples with low carrier density. We interpret the dependence of the contact resistivity on the carrier density as combination of thermionic field emission and of tunnelling transport. The former occurs between the crystallised layer and the metallisation and becomes relevant only at low carrier density. For our contacting procedure with ITO, we estimate a Schottky barrier of $0.75 \mathrm{eV}$. The latter occurs across the interfacial oxide between the wafer and the layer and dominates at high carrier density. By modelling 
the tunnelling transport, we can infer the band bending of the induced accumulation region and estimate that the areal density of charges trapped in the bulk or at the interfaces of the tunnelling oxide is in the low $10^{11} \mathrm{~cm}^{-2}$. For contacts fired in the optimum regime between 800 and $830^{\circ} \mathrm{C}$, we obtain contact resistivities between 20 and $50 \mathrm{~m} \Omega \mathrm{cm}^{2}$ which are uncritical for the fill factor in silicon solar cells.

VIII. OPEN ACCESS

The data underlying this contribution can be found under the following DOI: 10.5281/zenodo.3362368

\section{ACKNOWLEDGEMENT}

We thankfully acknowledge funding through the Horizon 2020 program of the European Union (EU) within the project DISC (GA No. 727539) and by the Swiss Federal Office for Energy (OFEN) within the project CHESS (Grant No. SI501253-01)

\section{REFERENCES}

[1] K. Yoshikawa, H. Kawasaki, W. Yoshida, T. Irie, K. Konishi, K. Nakano, T. Uto, D. Adachi, M. Kanematsu, H Uzu, and K. Yamamoto, 'Silicon heterojunction solar cell with interdigitated back contacts for a photoconversion efficiency over 26\%', Nature Energy, vol. 2, no. 5, p. 17032, May 2017, DOI: 10.1038/nenergy.2017.32.

[2] K. Yamamoto, K. Yoshikawa, H. Uzu, and D. Adachi, 'High-efficiency heterojunction crystalline Si solar cells', Jpn. J. Appl. Phys., vol. 57, no. 8S3, p. 08RB20, Jul. 2018, DOI: 10.7567/JJAP.57.08RB20.

[3] A. Richter, J. Benick, F. Feldmann, A. Fell, M. Hermle, and S. W. Glunz, 'n-Type Si solar cells with passivating electron contact: Identifying sources for efficiency limitations by wafer thickness and resistivity variation', Solar Energy Materials and Solar Cells, vol. 173, pp. 96-105, Dec. 2017, DOI: 10.1016/j.solmat.2017.05.042.

[4] F. Haase, C. Hollemann, S. Schäfer, A. Merkle, M. Rienäcker, J. Krügener, R. Brendel, and R. Peibst, 'Laser contact openings for local poly-Si-metal contacts enabling 26.1\%-efficient POLO-IBC solar cells', Solar Energy Materials and Solar Cells, vol. 186, pp. 184-193, Nov. 2018, DOI: 10.1016/j.solmat.2018.06.020.

[5] A. Tomasi, B. Paviet-Salomon, Q. Jeangros, J. Haschke, G. Christmann, L. Barraud, A. Descoeudres, J. P. Seif, S. Nicolay, M. Despeisse, S. De Wolf, and C. Ballif, 'Simple processing of back-contacted silicon heterojunction solar cells using selective-area crystalline growth', Nature Energy, vol. 2, no. 5, p. 17062, Apr. 2017, DOI: 10.1038 /nenergy.2017.62.

[6] News release - Imec and Jolywood achieve a record of 23.2 percent with bifacial n-PERT solar cells'. [Online]. Available: https://www.imec-int.com/en/articles/imec-andjolywood-achieve-a-record-of-23-2-percent-with-bifacialn-pert-solar-cells. [Accessed: 10-May-2019].

[7] A. Ingenito, G. Nogay, Q. Jeangros, E. Rucavado, C. Allebé, S. Eswara, N. Valle, T. Wirtz, J. Horzel, T. Koida, M. Morales-Masis, M. Despeisse, F.-J. Haug, P. Löper, and C. Ballif, 'A passivating contact for silicon solar cells formed during a single firing thermal annealing', Nature Energy, vol. 3, no. 9, pp. 800-808, Sep. 2018, DOI: 10.1038/s41560018-0239-4.
[8] S. Eidelloth and R. Brendel, 'Analytical Theory for Extracting Specific Contact Resistances of Thick Samples From the Transmission Line Method', IEEE Electron Device Letters, vol. 35, no. 1, pp. 9-11, Jan. 2014, DOI: 10.1109/LED.2013.2290602.

[9] D. K. Schroder, R. N. Thomas, and J. C. Swartz, 'Free Carrier Absorption in Silicon', IEEE Journal of Solid-State Circuits, vol. 13, no. 1, pp. 180-187, 1978, DOI: 10.1109/JSSC. 1978.1051012.

[10] M. A. Green, 'Self-consistent optical parameters of intrinsic silicon at $300 \mathrm{~K}$ including temperature coefficients', Solar Energy Materials and Solar Cells, vol. 92, no. 11, pp. 13051310, Nov. 2008, DOI: 10.1016/j.solmat.2008.06.009.

[11] D. Chandler-Horowitz and P. M. Amirtharaj, 'Highaccuracy, midinfrared $(450 \mathrm{~cm}-1 \leqslant \omega \leqslant 4000 \mathrm{~cm}-1)$ refractive index values of silicon', Journal of Applied Physics, vol. 97 , no. 12, p. 123526, Jun. 2005, DOI: 10.1063/1.1923612.

[12] D. Y. Smith, W. Karstens, E. Shiles, and M. Inokuti, 'Defect and analysis effects in the infrared optical properties of silicon', physica status solidi (b), vol. 250, no. 2, pp. 271277, Jan. 2013, DOI: 10.1002/pssb.201200495.

[13] M. A. Green, 'Intrinsic concentration, effective densities of states, and effective mass in silicon', Journal of Applied Physics, vol. 67, no. 6, pp. 2944-2954, Mar. 1990, DOI: 10.1063/1.345414.

[14] D. M. Riffe, 'Temperature dependence of silicon carrier effective masses with application to femtosecond reflectivity measurements', J. Opt. Soc. Am. B, JOSAB, vol. 19 , no. 5, pp. 1092-1100, May 2002, DOI: 10.1364/JOSAB.19.001092.

[15] G. L. Vick and K. M. Whittle, 'Solid solubility and diffusion coefficients of boron in silicon', Journal of the electrochemical society, vol. 116, no. 8, pp. 1142-1144, Aug. 1969, DOI: 10.1149/1.2412239.

[16] G. Masetti, M. Severi, and S. Solmi, 'Modeling of carrier mobility against carrier concentration in arsenic-, phosphorus-, and boron-doped silicon', IEEE Transactions on Electron Devices, vol. 30, no. 7, pp. 764-769, Jul. 1983, DOI: $10.1109 /$ T-ED.1983.21207.

[17] A. Mostafa and M. Medraj, 'Binary phase diagrams and thermodynamic properties of silicon and essential doping elements (Al, As, B, Bi, Ga, In, N, P, Sb and Tl)', Materials, vol. 10, no. 6, p. 676, Jun. 2017, DOI: 10.3390/ma10060676.

[18] M. A. Green, 'Accuracy of analytical expressions for solar cell fill factors', Solar Cells, vol. 7, no. 3, pp. 337-340, Dec. 1982, DOI: 10.1016/0379-6787(82)90057-6.

[19] A. Moldovan, F. Feldmann, K. Kaufmann, S. Richter, M Werner, C. Hagendorf, M. Zimmer, J. Rentsch, and M. Hermle, 'Tunnel oxide passivated carrier-selective contacts based on ultra-thin $\mathrm{SiO} 2$ layers grown by photo-oxidation or wet-chemical oxidation in ozonized water', in $42^{\text {nd }}$ IEEE Photovoltaic Specialist Conference (PVSC), Jun. 2015, pp. 1-6, DOI: 10.1109/PVSC.2015.7356144.

[20] A. Moldovan, F. Feldmann, M. Zimmer, J. Rentsch, J. Benick, and M. Hermle, 'Tunnel oxide passivated carrierselective contacts based on ultra-thin $\mathrm{SiO} 2$ layers', Solar Energy Materials and Solar Cells, vol. 142, pp. 123-127, Nov. 2015, DOI: 10.1016/j.solmat.2015.06.048.

[21] F. Feldmann, G. Nogay, P. Löper, D. L. Young, B. G. Lee, P. Stradins, M. Hermle, and S. W. Glunz, 'Charge carrier transport mechanisms of passivating contacts studied by temperature-dependent J-V measurements', Solar Energy Materials and Solar Cells, vol. 178, pp. 15-19, May 2018, DOI: 10.1016/j.solmat.2018.01.008.

[22] N. Folchert, M. Rienäcker, A. A. Yeo, B. Min, R. Peibst, and R. Brendel, 'Temperature-dependent contact resistance of carrier selective Poly-Si on oxide junctions', Solar Energy Materials and Solar Cells, vol. 185, pp. 425-430, Aug. 2018, DOI: 10.1016/j.solmat.2018.05.046. 
[23] H. C. de Graaff and J. G. de Groot, 'The SIS tunnel emitter: A theory for emitters with thin interface layers', IEEE Transactions on Electron Devices, vol. 26, no. 11, pp. 17711776, Nov. 1979, DOI: 10.1109/T-ED.1979.19684.

[24] A. Gehring and S. Selberherr, 'Modeling of tunneling current and gate dielectric reliability for nonvolatile memory devices', IEEE Transactions on Device and Materials Reliability, vol. 4, no. 3, pp. 306-319, Sep. 2004, DOI: 10.1109/TDMR.2004.836727.

[25] F. A. Padovani and R. Stratton, 'Field and thermionic-field emission in Schottky barriers', Solid-State Electronics, vol. 9, no. 7, pp. 695-707, Jul. 1966, DOI: 10.1016/00381101(66)90097-9.

[26] C. R. Crowell, 'The Richardson constant for thermionic emission in Schottky barrier diodes', Solid-State Electronics, vol. 8, no. 4, pp. 395-399, Apr. 1965, DOI: 10.1016/0038-1101(65)90116-4.

[27] A. G. Aberle, S. Glunz, and W. Warta, 'Impact of illumination level and oxide parameters on Shockley-ReadHall recombination at the $\mathrm{Si}-\mathrm{SiO} 2$ interface', Journal of Applied Physics, vol. 71, no. 9, pp. 4422-4431, May 1992, DOI: 10.1063/1.350782.

[28] T. Minami, T. Miyata, and T. Yamamoto, 'Work function of transparent conducting multicomponent oxide thin films prepared by magnetron sputtering', Surface and Coatings Technology, vol. 108-109, pp. 583-587, 1998, DOI: 10.1016/S0257-8972(98)00592-1.

[29] Y. Park, V. Choong, Y. Gao, B. R. Hsieh, and C. W. Tang, 'Work function of indium tin oxide transparent conductor measured by photoelectron spectroscopy', Applied Physics Letters, vol. 68, no. 19, pp. 2699-2701, 1996, DOI: $10.1063 / 1.116313$

[30] Y. Gassenbauer, R. Schafranek, A. Klein, S. Zafeiratos, M. Hävecker, A. Knop-Gericke, and R. Schlögl, 'Surface states, surface potentials, and segregation at surfaces of tin-doped $\mathrm{In}_{2} \mathrm{O}_{3}{ }^{\prime}$, Physical Review B, vol. 73, no. 24, p. 245312, 2006, DOI: 10.1103/PhysRevB.73.245312.

[31] F. G. Allen and G. W. Gobeli, 'Work Function, Photoelectric Threshold, and Surface States of Atomically Clean Silicon', Phys. Rev., vol. 127, no. 1, pp. 150-158, 1962, DOI: 10.1103/PhysRev.127.150.

[32] D. K. Schroder and D. L. Meier, 'Solar cell contact resistance-A review', IEEE Transactions on Electron Devices, vol. 31, no. 5, pp. 637-647, May 1984, DOI: 10.1109/T-ED.1984.21583. 